\title{
Validade do conceito de psicose processual ${ }^{1}$
}

\author{
Maurício Viotti Daker²
}

\begin{abstract}
Resumo: O conceito de processo, conforme Jaspers, em especial o de processo psíquico, é avaliado quanto à sua validade na psiquiatria atual. Após a definição do conceito, são revistas pesquisas sobre a evolução da esquizofrenia. Em seguida, são avaliados conjuntamente o conceito de processo psíquico, as evoluções da esquizofrenia e o próprio conceito de esquizofrenia. O processo psíquico é um conceito limite, uma ideia orientadora para o diagnóstico e que não deve ser dogmática. Ante a dificuldade de se definir a própria esquizofrenia, não nos parece um conceito datado, assim como não o são tantos outros conceitos na rica história de ideias da psiquiatria. Utilizado enquanto conceito limite, pode continuar a nos orientar diante de determinados casos de psicose. A dificuldade na definição do conceito de processo psíquico se encontra em sua localização intermediária na dicotomia mente-cérebro e a validade de seu conceito corresponde ao da validade da própria psiquiatria.
\end{abstract}

Palavras-chave: Processo psíquico; Esquizofrenia; Psicopatologia; Jaspers; Filosofia da mente.

\section{Validity of the concept of process psychosis}

Abstract: The concept of process as Jaspers, especially the psychic process, is evaluated for its validity in current psychiatry. After the definition of the concept, researches on the evolution of schizophrenia are reviewed. Then the concept of psychic process, the evolutions of schizophrenia and the concept of schizophrenia itself are evaluated jointly. The psychic process is a limit concept, a guiding idea for the diagnosis and that should not be dogmatic. Given the difficulty of defining schizophrenia itself, it does not seem to be a dated concept, so as many other concepts in the rich history of ideas of psychiatry. Used as a limit concept it can continue to guide us in front of particular cases of psychosis. The difficulty in defining the concept of psychic process is due to its

1 Trabalho apresentado no VI Painel de Psiquiatria da Associação Acadêmica Psiquiátrica de Minas Gerais - AAP-MG, federada da Associação Brasileira de Psiquiatria: "Desafios em Psiquiatria Forense". Conselho Regional de Medicina de Minas Gerais, Belo Horizonte, em 27 de abril de 2014.

2 Professor Associado do Departamento de Saúde Mental da FM-UFMG. Coordenador do Grupo de Investigação em Psicopatologia Conceitual, projeto de extensão da FM-UFMG. Doutor em medicina/psiquiatria pela Universidade de Heidelberg, CAPES/DAAD. 


\section{Validade do conceito de psicose processual}

intermediate location in the mind-body dichotomy and the validity of its concept corresponds to the validity of psychiatry itself.

Keywords: Psychic process; Schizophrenia; Psychopathology; Jaspers; Philosophy of mind

\section{Introdução}

O que é compreendido ou histórico-cultural seria redutível ao método explicativo natural, à abordagem causal? Ou o histórico-cultural e o natural seriam realidades diferentes? Ou, ainda, se fossem realidades indistintas, seriam acessíveis por métodos diferentes, o explicativo e o compreensivo? E será que necessitamos do método compreensivo, por questão prática, apenas enquanto a abordagem explicativa não dirimir todas as dúvidas? Ou, por questão de princípio, o método explicativo nunca esgotará o campo do compreensivo? A combinação de métodos, de qualquer forma, seria indispensável na psicologia e na psicopatologia?

As discussões e indagações acima eram candentes na época do jovem Jaspers, período caracterizado pelo Methodenstreit ou disputa de métodos (Jaspers, 1913; Daker, 2013). Ele frequentava as reuniões dominicais de intelectuais na residência de Weber em Heidelberg. Ao redigir sua Psicopatologia Geral (Allgemeine Psychopatologie), introduziu esse ideário na psiquiatria: “Ao invés de apresentar resultados dogmaticamente alegados, [o livro] pretende principalmente introduzir problemas, questionamentos, métodos; ao invés de um sistema baseado em uma teoria, pretende trazer uma ordem com base em reflexão metodológica" (Jaspers, 1913, p. VII).

Nesse contexto, surge o conceito de processo, bem como o de desenvolvimento, de forma e conteúdo, de delírio e ideias deliroides, o objeto e o todo do ser humano, enfim, o desdobrar do explicar e do compreender. 


\section{Maurício Viotti Daker}

\section{O que é processo?}

Caracteriza-se por mudança totalmente nova e duradoura na história da vida de uma pessoa, não compreendida empaticamente. Distingue-se, assim, de fase, bem como de crise (ou acesso) e de periodicidade (fases com certa uniformidade cíclica), igualmente não compreendidas empaticamente, mas que não são duradouras. E distingue-se do desenvolvimento de uma personalidade, que é o fluir gradual e compreensível empaticamente da história de vida, no contexto da evolução biológica normal.

O processo leva a uma transformação da personalidade. Esta poderá persistir assim alterada de forma estável (transformação que gera um novo estado) ou progredir continuamente até atingir um estado residual final. $\mathrm{O}$ processo pode ser insidioso ou surgir em brotos ou surtos, que são processos agudos, tempestuosos, que vão levando à transformação da personalidade. Nada impede que fases e reações possam ocorrer nos intervalos entre os surtos e, em alguns casos, serem confundidas com estes (Jaspers, 1946, p. 581).

Notam-se, ao longo das edições da Psicopatologia Geral de Jaspers, acréscimos conceituais que envolvem a noção de processo (Tripicchio, 2008). Em especial, a constatação do que seria o processo normal, ou seja, no contexto da evolução biológica da vida, das mudanças biológicas esperadas em cada idade do indivíduo (podem ocorrer também fases e acessos). No processo normal, diferentemente do patológico, é preservada a unidade da personalidade que transcende todas as etapas etárias da vida. Já no patológico, há destruição dessa unidade, perde-se a continuidade de sentido da evolução da história da vida.

Há dois tipos de processo patológico: o orgânico e o psíquico. O orgânico diz respeito às doenças conhecidas (e talvez a alguns casos do grupo da demência precoce, como menciona Jaspers) que acometem o cérebro e levam a uma destruição geralmente grosseira do psiquismo, a um emaranhado de sintomas ao acaso. É admitida a remissão ou cura em alguns casos. O processo psíquico, sem uma causa ou acometimento cerebral conhecido, é uma alteração da personalidade sem aquela destruição mais grosseira do psiquismo e com conexões evolutivas psicológicas típicas a que temos acesso, e que seria pouco 


\section{Validade do conceito de psicose processual}

mais compreensível em relação às manifestações do processo orgânico (no tocante à situação, meio e vivência relacionados ao conteúdo, não à sua gênese) e é considerado irreversível (Jaspers faz analogia com o envelhecimento, que não tem retorno). Corresponde a muitos casos da esquizofrenia. Postula-se que deva haver acometimento orgânico ou biológico desconhecido no processo psíquico, mas este envolveria muito mais do que simplesmente um acometimento orgânico (Jaspers, 1946, p. 581-593).

Vale lembrar que, na clínica, um acometimento orgânico ou tóxico conhecido (dito exógeno) pode precipitar em pessoa vulnerável alteração da personalidade com "conexões evolutivas psicológicas típicas" (seriam ou incluiriam complexos de sintomas típicos da esquizofrenia, ditos endógenos), sem que o acometimento orgânico propriamente as tenham originado. Para aprofundamento no tocante ao exógeno e ao endógeno, referimos a recente artigo de Messas (2013).

Jaspers observa que o conceito de processo psíquico é um conceito limite e não um conceito de espécie bem delimitável e definível, e que, em vez de “processo psíquico", poder-se-ia denominá-lo de "evento biológico total". Aqui, entende-se por "biológico" o conceito amplo de totalidade da vida e não as manifestações parciais desta, somáticas ou psíquicas. "As palavras expressam o enigma, mas não o esclarecem" (Jaspers, 1946, p. 582).

É certo que o conceito de processo psíquico espelha, em grande medida, a definição de demência precoce de Kraepelin, mantida certa cautela quanto às possíveis causas orgânicas:

Sob o nome de dementia praecox, seja-nos permitido resumir, provisoriamente, uma série de quadros de doenças cuja propriedade comum consiste no desfecho em peculiar estado deficitário. Parece, na verdade, que esse desfecho desfavorável 


\section{Maurício Viotti Daker}

não tenha que ocorrer sem exceção, mas ocorre com tal frequência que, por enquanto, pretendemos insistir na denominação em uso. [...] Com base nos fatos clínicos e anatômicos até agora conhecidos, não posso duvidar de que lidamos aqui com lesões graves do córtex cerebral e, de regra, no máximo parcialmente reversíveis. No entanto, se o processo de doença é sempre o mesmo, isso, por ora, tem que ser considerado ainda totalmente incerto. (Daker, 1999)

\section{O que dizem as pesquisas recentes sobre a evolução da esquizofrenia?}

As pesquisas replicam, em linhas gerais, as observações de Kraepelin, inclusive no tocante às exceções quanto ao curso inexorável a um estado deficitário cognitivo e afetivo, que lembra uma demência. Mesmo porque estes seriam achados, em certa medida, tautológicos, ou seja, uma vez definida a esquizofrenia com base em seu curso desfavorável, é previsível que as pesquisas indicarão esse curso: são excluídos das pesquisas quadros que não preenchem as definições vigentes, embora possam se relacionar à esquizofrenia.

Estudos catamnésticos dos mais consistentes foram os de Ciompi e de Huber, com grande número de pacientes e tempo longo de seguimento (Ciompi, 1980; Huber, 1980). O gráfico 1 mostra os desfechos evolutivos encontrados por Ciompi e Huber que, no geral, corroboram dados anteriores igualmente consistentes de Manfred Bleuler. Note-se que a evolução favorável, conforme aceito atualmente, gira também em torno de 20\% (DSM-5, 2013, p. 102). A figura 1 e a tabela 1 mostram os vários tipos de evolução reconhecidos por Ciompi e Huber, respectivamente (na figura 1 não constam cerca de 20\% dos casos que concluíram o estudo, pois suas evoluções foram indeterminadas). Como se vê, Ciompi foi capaz de distinguir oito tipos de evolução (figura 1) e Huber chegou a distinguir 12 tipos (Huber, 1980). 
Validade do conceito de psicose processual

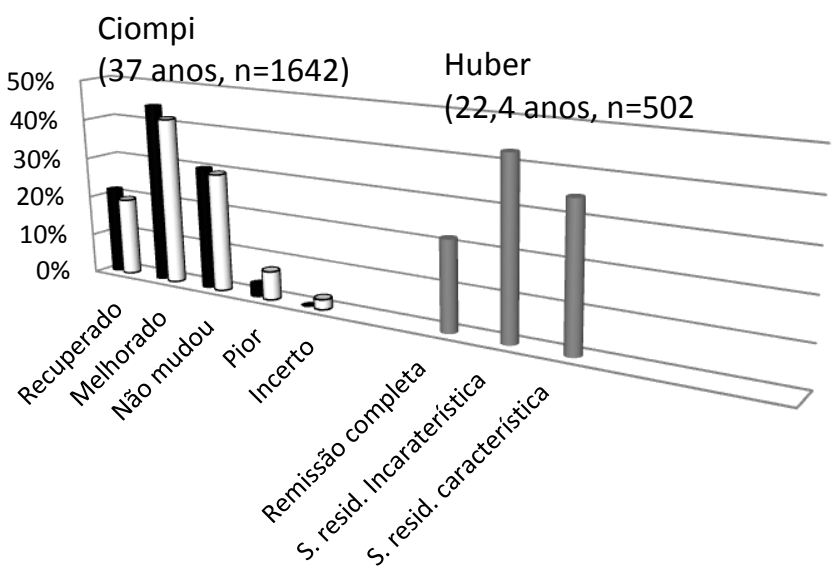

- Mulheres (Ciompi) $\quad \square$ Homens (Ciompi) ambos sexos (Huber)

Gráfico 1: Desfecho da esquizofrenia, segundo Ciompi e Huber.

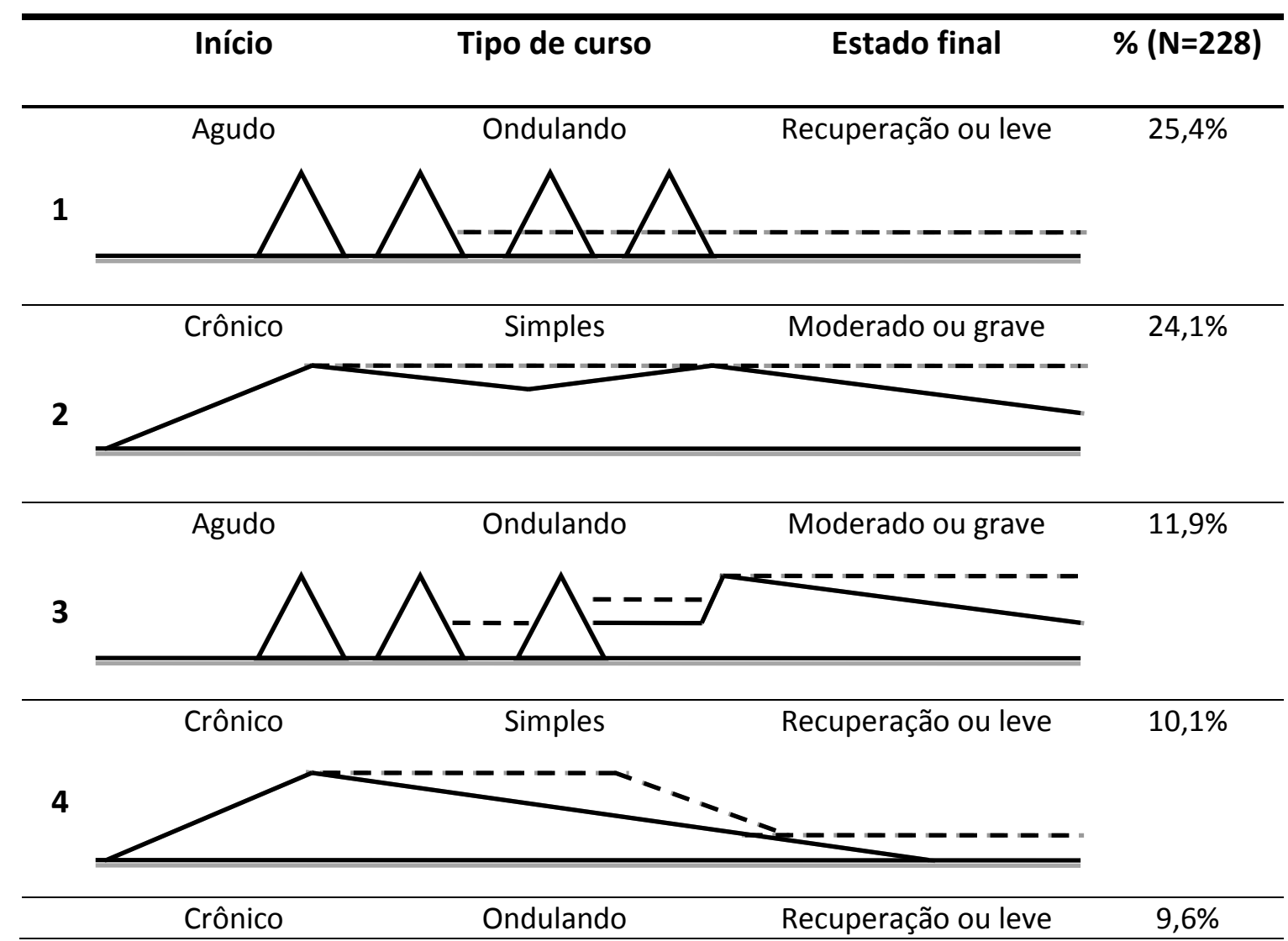

Psicopatologia Fenomenológica Contemporânea, 2014, 3 (2), 1-15 


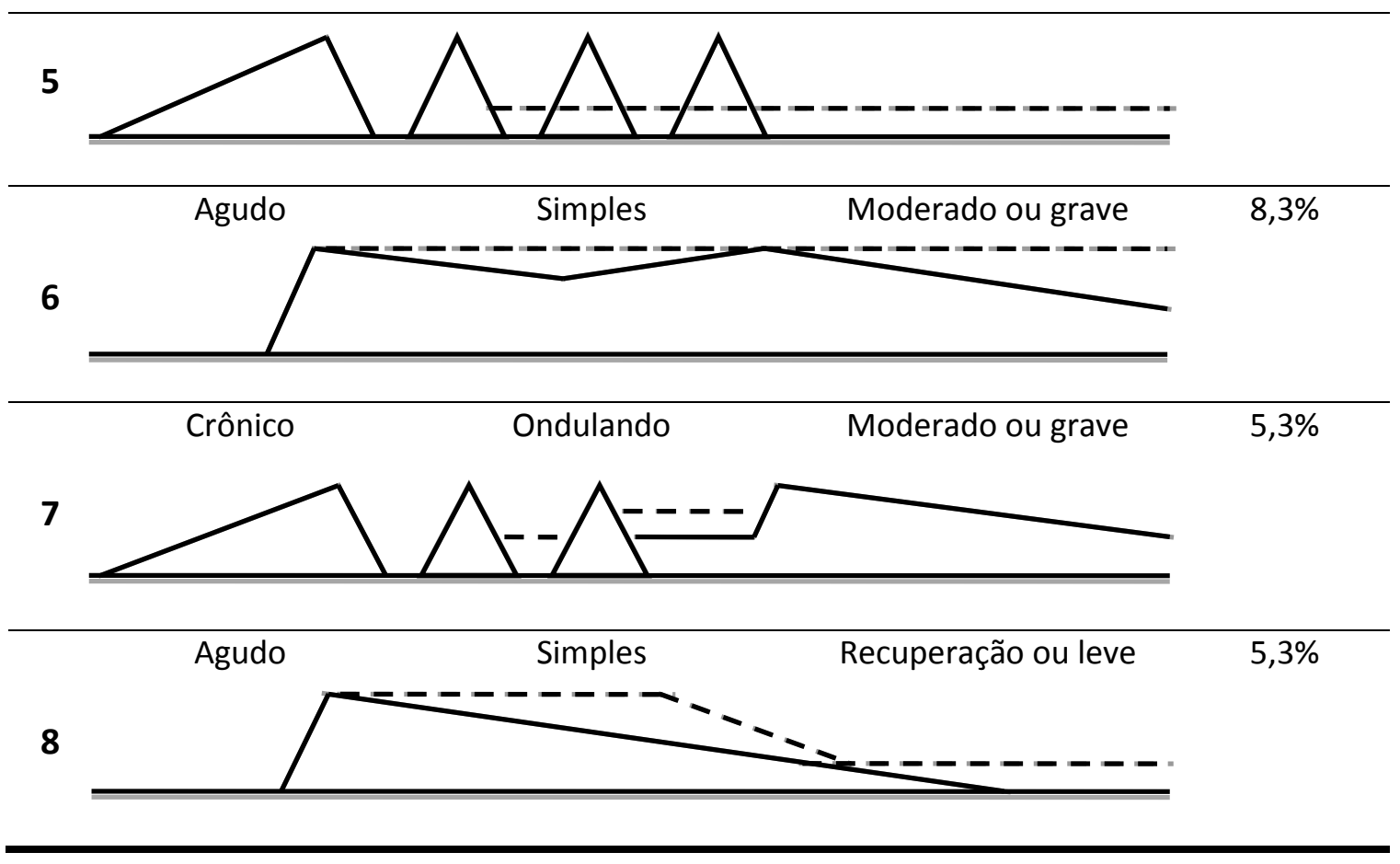

Figura 1: Representação esquemática de tipos de curso (Ciompi, 1980).

\begin{tabular}{|c|c|c|}
\hline Tipo de desfecho & $\mathrm{N}=502$ & $\begin{array}{c}\text { Grupos de tipos de } \\
\text { desfecho }\end{array}$ \\
\hline Remissão completa & $\begin{array}{c}111 \\
22,1 \%\end{array}$ & $\begin{array}{c}111=22,1 \% \\
\text { Remissão completa }\end{array}$ \\
\hline Síndromes residuais puras & $\begin{array}{c}202 \\
40,2 \%\end{array}$ & \multirow{2}{*}{$\begin{array}{c}217=43,2 \% \\
\text { Síndromes residuais não } \\
\text { características }\end{array}$} \\
\hline Deformidade estrutural sem psicose & $\begin{array}{c}15 \\
3,0 \%\end{array}$ & \\
\hline Síndromes residuais mistas & $\begin{array}{c}83 \\
16,5 \%\end{array}$ & \multirow{4}{*}{$\begin{array}{c}174=34,7 \% \\
\text { Síndromes residuais } \\
\text { características }\end{array}$} \\
\hline Psicose com típico defeito esquizofrênico & $\begin{array}{c}54 \\
10,8 \%\end{array}$ & \\
\hline Psicose pura crônica & $\begin{array}{c}21 \\
4,2 \% \\
\end{array}$ & \\
\hline Deformidade estrutural com psicose & $\begin{array}{c}16 \\
3,2 \% \\
\end{array}$ & \\
\hline
\end{tabular}

Tabela 1: Tipos de desfecho após média de 22,4 anos de enfermidade (Huber, 1980). 


\section{Validade do conceito de psicose processual}

Observa-se que a evolução da esquizofrenia é muito heterogênea e bastante imprevisível em cada caso. Geralmente, sabe-se que o início insidioso indica prognóstico ruim e o início agudo prognóstico melhor, mas estas estão longe de serem regras gerais, haja vista as evoluções 3, 4, 5 e 6 da figura 1. A evolução 6, de início agudo e curso sempre grave, corresponde à antiga esquizofrenia catastrófica, termo utilizado por Manfred Bleuler, cuja prevalência teria se reduzido devido às novas modalidades terapêuticas, fato que pode ter interferido na evolução da esquizofrenia como um todo.

Vários estudos mais recentes corroboram a complexa variabilidade dos cursos da esquizofrenia e contradizem a tendência de alguns autores à dicotomização entre um curso benigno com bom prognóstico e outro crônico deteriorante. Valliant (1978) reviu 51 pacientes de um trabalho anterior, de 1964, que tinham sido considerados de bom prognóstico e com remissão completa. Cerca de $40 \%$ deles se tornaram crônicos, mostrando a dificuldade de se prever o curso desde o início: os que cronificaram tinham praticamente a mesma apresentação daqueles que se mantiveram em remissão. Fenton e McGlashan (1987), em suas conclusões, refletem o habitual tom pessimista quanto à evolução da esquizofrenia: características de mau prognóstico praticamente garantem prognóstico pobre, enquanto que características de bom prognóstico não garantem bom prognóstico.

Quanto mais tempo se acompanha a evolução, observam-se naturalmente menos daqueles episódios únicos iniciais que continuam em remissão: 50,3\% em 2 anos de acompanhamento (Jablensky et al., 1992), 22\% em 5 anos (Shepherd et al., 1989), 18\% em 13 anos (Mason et al., 1997), 12,2\% em 15 anos (Wiersma et al., 1998). Wiersma et al. (1998) concluíram que uma grande maioria da amostra que recaiu o fizeram até os 5 anos ( $70 \%$ do total) e que o risco de recaída parecia desaparecer após 9 anos. Jackson e Birchwood (1996) 


\section{Maurício Viotti Daker}

concluíram que a deterioração ocorre na fase pré-psicótica e inicial da doença, mas que usualmente se estabiliza por volta dos 5 anos.

Rosen e Garety (2005), em curso de 6 anos, confirmaram a heterogeneidade da evolução e distinguiram cinco grupos: episódio único sem sintomas persistentes $(16,5 \%)$, episódios múltiplos sem sintomas persistentes (36\%), episódios múltiplos com sintomas persistentes e sem retorno à normalidade $(29,5 \%)$ e episódio único com sintomas persistentes e sem retorno à normalidade $(6,5 \%)$, além de grupo de curso indeterminado $(9,5 \%$, e de cerca de $2 \%$ de óbito). Identificaram nove variáveis que distinguiam, de modo estatisticamente significante, pacientes que tiveram apenas 1 episódio sem sintomas persistentes versus o restante dos pacientes: sexo, estado civil, status de emprego, ajustamento pré-mórbido, insight, relato de ter usado drogas antes ou durante o episódio, duração do pródromo, duração do pródromo combinada com duração de psicose não tratada e duração do primeiro episódio. Porém, essas variáveis não são significantes para distingui-los do grupo seguinte de episódios múltiplos sem sintomas persistentes (salvo no quesito insight, como seria de se esperar para pacientes com um episódio versus os de múltiplos episódios), mais uma vez contradizendo possível dicotomia entre quadros deteriorantes e benignos.

Acrescentem-se a essa heterogeneidade evolutiva as diferenças socioculturais, como a observada entre países desenvolvidos e em desenvolvimento no International Pilot Study for Schizophrenia (Sartorius et al., 1977) e International Study of Schizophrenia (Jablensky et. al, 1992). Maior contato social com família e amigos é fator positivo para prevenir recaídas (Jablensky et al., 1992; Carpenter e Strauss, 1991), o que poderia se correlacionar com a pior evolução em países desenvolvidos. Mas a natureza desse contato com a família é importante, pois ambientes muito críticos ou com elevadas emoções expressas atuam negativamente. Segundo Harrison et al. (2001), conforme o follow up do International Study of Schizophrenia, é possível que casos que não remitiam no início atinjam recuperação em fase tardia. Por outro lado, a resposta ao tratamento nos primeiros 2 anos (menor período de tempo com sintomas 


\section{Validade do conceito de psicose processual}

psicóticos nesse período) foi o melhor preditor de desfecho aos 15 anos de seguimento, fato que indica a importância do tratamento precoce.

É geralmente aceito que, no início, o quadro é mais mutável e, após 5 anos, mais estável. Por estabilidade em psiquiatria, entende-se: o paciente pode permanecer no atual ambiente em que vive, não há piora de sintomas psiquiátricos que ameacem a habilidade do paciente de manter-se funcional no atual nível, ausência de sintomas psiquiátricos que levem a riscos para si ou para terceiros, ausência de condição médica potencialmente letal, não há mudança no suporte psicossocial ou no acesso ao tratamento que ameacem a habilidade de continuar como acima (Weiden et al., 2007).

Somando-se os conhecidos fatores de bom e mau prognóstico da esquizofrenia, o melhor ou pior prognóstico conforme o conceito de esquizofrenia que se tem, mais a noção de estabilidade, resultarão duas tendências terapêuticas básicas: modelo de manutenção ou modelo de recuperação (Weiden et al., 2007). Ante a estabilidade obtida com o tratamento, os pessimistas quanto à evolução da esquizofrenia se darão por contento com a estabilidade atingida, em atitude conservadora. Já os mais otimistas procurarão a remissão, ainda que a estabilidade obtida possa ser colocada em risco.

\section{Processo, evolução e conceito da esquizofrenia}

Como ficaria o conceito jasperiano de processo defronte a essas pesquisas sobre a evolução da esquizofrenia? A julgar pelo fato de que o próprio Kraepelin e, por conseguinte, Jaspers, dentre outros, já identificavam casos de remissão da demência precoce, não haveria razão, nesse aspecto, para tornar o conceito ultrapassado. Por outro lado, os estudos indicam que a maioria dos esquizofrênicos não se recupera totalmente, ainda que submetidos às novas modalidades terapêuticas, o que condiz com o conceito de processo psíquico. Como Jaspers costumava dizer, em tom kantiano, não se deve confundir uma 


\section{Maurício Viotti Daker}

ideia com os fatos. Processo é um conceito, uma ideia que nos serve de orientação diagnóstica, não que tenha que se aplicar sempre e em qualquer lugar para os quadros clínicos reais, muito menos que se torne dogmático. Como dito, é uma noção limite. Essa visão relativa, não absoluta e sem recair em veredito estático ou em um tudo ou nada, propicia abordagem maleável, não engessada, do processo esquizofrênico, com a possibilidade de elaboração de estratégias terapêuticas onde há genuínas perspectivas de mudança (Tamelini, 2013).

Se considerarmos o conceito bleuleriano amplo de esquizofrenia, por demais citado e conhecido por Jaspers, e em que pese a negação do restitutio ad integrum (Bleuler, 1950, p. 9), veremos que a esquizofrenia se dilui nos quadros latentes e nas pessoas esquizoides. Digno de nota, tal esquizofrenia latente esteve por ressuscitar no DSM-5 sob a designação de "síndrome de psicose atenuada", mas que foi alocada no apêndice "condições para estudo posterior" (DSM-5, p. 783-86) por contendas médicas, legais e sociais. O DSM-5 tem abordado vários transtornos em termos de espectros, em que transtornos diferentes são agregados devido à possibilidade de compartilharem elementos comuns. Alguns mais graves e outros menos, sendo que, além da transição entre eles, a diferenciação entre o normal e o patológico no âmbito dos menos graves também se torna difícil, o que não é nenhuma novidade na psiquiatria.

Dividimo-nos entre o conceito nuclear original do jovem Kraepelin (ele mesmo questionou sua concepção mais tarde), o de uma doença de mau prognóstico, para a qual haveria de se encontrar uma causa específica cerebral, e a noção abrangente de um espectro contínuo, tanto em direção aos transtornos de humor e a outros transtornos mentais quanto à normalidade. Os limites entre umas coisas e outras são, em muitos casos, como diria Janzarik (1980), convenções.

A rigor, o conceito de esquizofrenia não está bem estabelecido com base em evidências científicas, de modo que não se sabe se é uma entidade nosológica própria, se várias doenças acobertadas por uma grande 


\section{Validade do conceito de psicose processual}

manifestação sindrômica, se diluída em outros transtornos mentais e mesmo na normalidade psíquica, ou se um tanto de tudo isso.

Assim sendo, podemos definir uma esquizofrenia processual (processo psíquico) para aqueles casos em que não mais haveria retorno à normalidade (não que melhoras parciais inexistam). Seriam as esquizofrenias geralmente mais graves e comprometedoras. Ainda assim, segundo o próprio conceito de Jaspers, o processo pode se arrefecer e estabilizar em formas mais brandas. Haveria então uma esquizofrenia processual de evolução progressiva até um estado residual e outras de evolução incompleta até certo platô de estabilidade.

Resta a questão de até onde a esquizofrenia seria compreensível, e se melhor delimitável em parâmetros não estatísticos, temas estes candentes na psicopatologia fenomenológica contemporânea.

\section{Conclusões}

Qual a validade atual do conceito de processo, em especial do processo psíquico? Não nos parece um conceito datado, ou seja, de valor apenas histórico. Permanece atual e útil, como costuma ocorrer com muitos dos conceitos empregados ao longo da rica história de ideias da psiquiatria e da psicopatologia. O processo orgânico não parece gerar maiores discussões, nem os aspectos psíquicos no desenvolvimento de uma personalidade, que espelham a bem conhecida e facilmente assimilável dicotomia corpo-alma. $\mathrm{O}$ problema é quando confluem corpo e alma, cérebro e mente. O processo psíquico quebra essa dicotomia e se coloca ali onde o paciente psiquiátrico e a psiquiatria se encontram, na confluência entre mente e cérebro. A validade do processo psíquico, portanto, coaduna-se com a validade da própria psiquiatria. 


\section{Maurício Viotti Daker}

\section{Referências bibliográficas}

American Psychiatric Association (2013). Diagnostic and statistical manual of mental disorders. $5^{\text {th }}$ ed. Arlington, VA: American Psychiatric Association.

Bleuler, E. (1950). Dementia praecox or the group of schizophrenias. New York: International University Press.

Carpenter W.T., \& Strauss J.S. (1991). The prediction of outcome in schizophrenia: IV. Eleven-year follow-up of the Washington IPSS cohort. Journal of Nervous and Mental Disease, 179, 517-525.

Ciompi C. (1980). Catamnestic long-term study on the course of life and aging of schizophrenics. Schizophrenia Bulletin, 6(4), 606-618.

Daker M.V. (1999). Demência precoce na sexta edição de Kraepelin em 1899. Casos Clin Psiquiatr, 1(1), 59-67.

Daker M.V. (2013). Filosofia e Psiquiatria. In R.M. Oliveira (Org.), Seminários em psicopatologia: da psiquiatria clássica à contemporaneidade (pp. 57-65). Belo Horizonte: Coopmed.

Fenton W.S., \& McGlashan T.H. (1987). Prognostic scale for chronic schizophrenia. Schizophrenia Bulletin, 13(2), 277-286.

Fulford B., Thorton T., \& Graham G. (2006). Psychopathology and the 'Methodenstreit'. In B. Fulford, T. Thorton \& G. Graham (Org.), Oxford textbook of philosophy and psychiatry (pp. 211-238). Oxford, New York: Oxford University Press.

Harrison G., Hopper K., Craig T., Laska E., Siegel C., Wanderling J., ... Wiersma D. (2001). Recovery from psychotic illness: a 15-year and 25-year international follow-up study. British Journal of Psychiatry, 178, 506517. 
Huber G., Gross G., Schüttler R., \& Linz M. (1980) Longitudinal studies of schizophrenic patients. Schizophrenia Bulletin, 6(4), 592-605.

Jablensky A., Sartorius N., Ernberg G., Anker M., Korten A., Cooper J.E., ... Bertelsen A. (1992). Schizophrenia: Manifestations, incidence and course in different cultures. A World Health Organization tencountry study. Psychological Medicine,20(Suppl), 1-97.

Jackson C., \& Birchwood M. (1996). Early intervention in psychosis: Opportunities for secondary prevention. British Journal of Clinical Psychology, 35, 487-502.

Janzarik W. (1980). Der schizoaffecktive Zwischenbereich und die Lehre von den primären und sekundären Seelentörungen. Nervenarzt, 51(5), 272-279.

Jaspers K. (1913). Allgemeine Psychopathologie. Berlin: Julius Springer.

Jaspers K. (1946). Allgemeine Psychopathologie. $4^{\mathrm{a}}$ ed. Berlin, Heidelberg: Springer.

Mason P., Harrison G., Croudace T., Glazebrook C., \& Medley I. (1997). The predictive validity of a diagnosis of schizophrenia. A report from the International Study of Schizophrenia (ISoS) coordinated by the World Health Organization and the Department of Psychiatry, University of Nottingham. British Journal of Psychiatry, 170, 321-327.

Messas G.P. (2013) Sentido e limites do diagnóstico diferencial entre psicoses endógenas e exógenas. Psicopatologia Fenomenológica Contemporânea, 2(1), 2-15.

Rosen K., \& Garety P. (2005). Predicting recovery from schizophrenia: a retrospective comparison of characteristics at onset of people with single and multiple episodes. Schizophrenia Bulletin, 31, 735-750. 


\section{Maurício Viotti Daker}

Sartorius N., Jablensky A., \& Shapiro R. (1997). Two-year follow-up of the patients included in the WHO International Pilot Study of Schizophrenia: Preliminary communication. Psychological Medicine, 7, 529-541.

Shepherd M., Watt D., Falloon I.R.H., \& Smeeton N. (1989) The natural history of schizophrenia: a five-year follow-up study of outcome and prediction in a representative sample of schizophrenics. Cambridge, UK: Cambridge University Press.

Tamelini M.G. (2013). O processo psíquico sob a ótica fenomenológica. Psicopatologia Fenomenológica Contemporânea, 2(1), 91-102.

Tripicchio A. (2008) Processo e desenvolvimento de Karl Jaspers. Reflexões sobre o campo psi. Recuperado de <http://www.redepsi.com.br/2008/01/20/processo-edesenvolvimento-de-karl-jaspers/>.

Vaillant G.E. (1978). A 10-year follow- up of remitting schizophrenics. Schizophrenia Bulletin, 4(1), 78-85.

Weiden P.J., Preskorn S.H., Fahnestock P.A., Carpenter D, Ross R, \& Docherty JP. (2007). Translating the Psychopharmacology of Antipsychotics to Individualized Treatment for Severe Mental Illness: A Roadmap. J Clin Psychiatry, 68 (suppl 7), 1-48.

Wiersma D., Nienhuis F.J., Slooff C.J., \& Giel R. (1998). Natural course of schizophrenic disorders: A 15-year follow-up of a Dutch incidence cohort. Schizophrenia Bulletin, 24(1), 75-85. 